\title{
New Developments in IR Lock-in Thermography
}

\author{
O. Breitenstein, J.P. Rakotoniaina, M. Hejjo Al Rifai, and M. Gradhand \\ Max Planck Institute of Microstructure Physics, Weinberg 2, D-06120 Halle, Germany \\ breiten@mpi-halle.de, +49-345-5582740 \\ F. Altmann and T. Riediger \\ Fraunhofer Institute for Mechanics of Materials, Heideallee 19, D-06120 Halle, Germany \\ fa@iwm-fhg.de, +49-345-5589139
}

\section{Introduction}

Lock-in thermography based on an infrared (IR) camera has proven to be a useful tool for failure analysis of integrated circuits (ICs) [1,2]. This technique is based on the application of a supply voltage to the IC which is periodically pulsed in the $3 \mathrm{~Hz}$ to $1 \mathrm{kHz}$ range. Any faults dissipating electrical energy lead to periodically oscillating local heat sources, which cause a periodic temperature (T-) modulation at the surface. These heat sources are imaged by the IR camera with its output digitally on line lock-in processed to yield the amplitude and phase of the local T-modulation. The sensitivity of this a.c. technique can be considerably better than that of any other direct thermal imaging technique due to its averaging nature and suppression of the steady-state IR image. Nevertheless, the T-modulation amplitude images are modulated by the IR emissivity contrast, which may complicate the interpretation of the images. However, this can be avoided by displaying the phase image $[1,2]$. Thus, lock-in thermography has the potential to considerably expand the application field of thermal failure analysis. In this contribution four new technical developments of lock-in thermography will be introduced. These developments are (1) blackening the IC surface with colloidal bismuth, (2) the synchronous undersampling technique allowing the use of higher lock-in frequencies, (3) displaying the $0^{\circ} /-90^{\circ}$ signal as a novel high resolution emissivity corrected image type, and (4) removing the thermal blurring effect by mathematically deconvoluting the $0 \%-90^{\circ}$ signal. The effect of these techniques is demonstrated by using a regularly working operational amplifier ( $\mu \mathrm{A}$ 741) and a damaged capacitor as test devices. It will be shown that blackening the IC surface improves the detection sensitivity in metallized regions by up to a factor of 10 , whereas the other methods allow improvement of the effective spatial resolution. We will also discuss which of the spatial resolution improvement techniques is most appropriate in different situations.

\section{Methods and Analysis}

\section{Blackening the surface of ICs}

The thermal radiation signal detected by the IR camera is generally the product of the temperature dependent black body radiation and the value of the local IR emissivity $\varepsilon$, which equals the absorbance and is always less than $100 \%$ [2]. Metallized layers in particular show a very low emissivity in the range of 1 to $5 \%$ and therefore usually appear dark in the IR image, even in an unpowered device. This phenomenon is called "emissivity contrast". Therefore in a single IR image (topography image) weak heat sources can hardly be recognized since the emissivity contrast is larger than the thermal contrast. It is one of the basic advantages of lock-in thermography that, by displaying the phase signal instead of the amplitude signal, the IR emissivity contrast is effectively suppressed $[1,2]$. The mathematical reason for this is that the phase signal relies on the quotient between the out-of-phase signal $\left(-90^{\circ}\right.$ signal $)$ and the in-phase signal $\left(0^{\circ}\right.$ signal $)$, which are both linearly proportional to the local IR emissivity $\varepsilon$. However, if a heat source is located below a metallized layer having low emissivity, its IR signal will be considerably less and may approach the noise level of the measurement setup. Thus, the effective detection sensitivity is usually lowered by a factor of 10 for heat sources beneath metallized regions. This may prevent the detection of these weak heat sources. The usual way to prevent this effect in conventional IR thermography is to cover the surface with a thin layer which is "black" in the wavelength range of interest (3 to $5 \mu \mathrm{m}$ ). Such an IR emitter layer should be essentially structureless within the best possible spatial resolution of about $5 \mu \mathrm{m}$, should be electrically insulating in order not to short-circuit the device and the current leads, and should be thin enough in order not to excessively degrade the spatial resolution (max. $10 \mu \mathrm{m})$. Paints hardly fulfill all these requirements. Colloidal gold is often used as a thin IR absorber for constructing thermal IR detectors. However, colloidal gold is electrically conductive. We have found that colloidal bismuth, evaporated at a residual air pressure of $200 \mathrm{~Pa}$ in a closed evaporation chamber, fulfills these requirements best. This process results in a black layer that has a thickness of about 10 microns and consists from 
nanoscopic flakes which can be easily and completely removed by immersing the device for some seconds in an ultrasonic water bath.

\section{Synchronous undersampling}

The lock-in frequency $f_{\text {lock-in }}$ is an important parameter for lockin thermography, since it influences the thermal diffusion length [2]. Higher $f_{\text {lock-in }}$ results in better effective spatial resolution, hence reducing the influence of the inevitable thermal blurring effect. On the other hand, a high value of $f_{\text {lock-in }}$ also leads to lower signal strength, especially for extended heat sources. Until now, $\mathrm{f}_{\text {lock-in }}$ was limited by the frame rate $\mathrm{f}_{\text {frame }}$ of the IR camera to $f_{\text {lock-in }}<f_{\text {frame }} / 4$, since, according to the Nyquist principle, at least 4 frames must be captured in each lock-in period to yield the amplitude and phase information in each position independently. If more than the 4 necessary images are captured, one speaks of "oversampling". However, it is known from the application of lock-in thermography in nondestructive testing that $f_{\text {ock-in }}$ may also be allowed to come close to $f_{\text {frame }}$ and may even exceed $f_{\text {frame }}$ if the measurement is evaluated over a sufficiently large number of periods [3]. In that case the measurements in different phase positions are taken in different successive lock-in periods. This so-called "undersampling" technique usually operates without any synchronization between $f_{\text {lock-in }}$ and $f_{\text {frame }}$. However, this may lead to interference at certain "collision" lock-in frequencies thus disturbing the measurement [3]. To avoid this, undersampling can also be used in a synchronously working system if $\mathrm{f}_{\text {lock-in }}=\mathrm{f}_{\text {frame }}(\mathrm{m}+1 / 4)$ holds exactly, with " $\mathrm{m}$ " the order of undersampling being an integer greater than zero [2]. However, this type of operation is technically difficult to realize with the lock-in thermography system. Therefore we have developed an alternative timing procedure for the TDL $384 \mathrm{M}$ 'Lock-in' system [4] which is shown in Fig. 1 together with the usual lock-in timing sequence and the synchronous undersampling procedure mentioned above for $\mathrm{m}=1$.

a)



b)

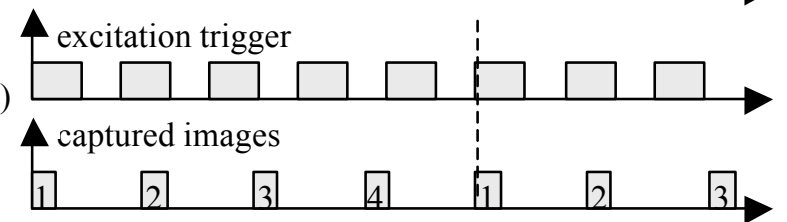

c)

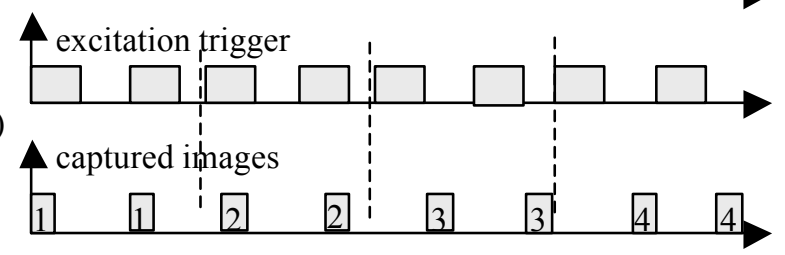

Figure 1: Timing schemes of three lock-in thermography procedures: Conventional lock-in procedure with 4 frames per lock-in period belonging to 4 different phase positions of the excitation trigger (a), the conventional synchronous undersampling procedure for $m=1$ (b), and the newly proposed undersampling procedure (c). Shown in (c) is the sequence for first order undersampling. For higher order undersampling the lock-in trigger frequency may be a multiple of the frame rate.

In the usual synchronous lock-in timing sequence (a) 4 frames belonging to 4 different phase positions of the excitation trigger $\left(1,2,3\right.$, and 4 ; corresponding to $0^{\circ}, 90^{\circ}, 180^{\circ}$, and $\left.270^{\circ}\right)$ are captured within one lock-in period and separately summed for subsequent measurements in 4 different frame storages. These 4 images are the basis of the lock-in correlation procedure $[2,3]$. The end of one complete cycle is indicated in Fig. 1 by a vertical dashed line.

In the conventional synchronous undersampling procedure (b) the measurements belonging to different phase positions are measured one after the other in subsequent excitation trigger periods. In the new procedure (c) the lock-in frequency exactly equals (or is a multiple of) the frame rate of the IR camera. By using conventional TTL-logic, the phase difference between the frame rate and the lock-in trigger can be set constant to $0^{\circ}$, $90^{\circ}, 180^{\circ}$, or $270^{\circ}$. Note: Fig. $1 \mathrm{~b}$ shows only two measurements per phase setting, but in reality there may be many more.

In procedure (c) all measurements belonging to phase position 1 are averaged first, then the phase is switched to position 2 and the same number of images is averaged, and so on. This timing sequence is much easier to realize than that of Fig. 1 (b). We have developed a PLL-driven undersampling trigger generator allowing for $\mathrm{m}=1,2,4$, and 8 . Hence for $\mathrm{f}_{\text {frame }}=120$ $\mathrm{Hz}$ possible undersampling lock-in frequencies are $120 \mathrm{~Hz}, 240$ $\mathrm{Hz}, 480 \mathrm{~Hz}$, and $960 \mathrm{~Hz}$. If the IR camera is not running in full frame mode but instead only a selected range of the detector is being used, the frame rate increases up to $350 \mathrm{~Hz}$ for an image of $128 \times 128$ pixels. Then the maximum possible lock-in frequency increases to $8 \times 350 \mathrm{~Hz}=2.8 \mathrm{kHz}$. This new undersampling system is now optionally available for the TDL 384 M 'lock-in' thermography system [4].

\section{Display of the $0^{\circ} /-90^{\circ}$ signal}

It has already been mentioned that the phase signal $\Phi(\mathrm{x}, \mathrm{y})$, is free of the emissivity contrast since it is actually a measure of the time delay of the local T-modulation with respect to the pulsed bias applied to the sample. This signal is calculated from the measured in-phase signal $S^{\circ}(x, y)$ and out-of-phase signal $\mathrm{S}^{-90^{\circ}}(\mathrm{x}, \mathrm{y})$ as:

$$
\Phi(\mathrm{x}, \mathrm{y})=\arctan \left(\frac{-\mathrm{S}^{-90^{\circ}}(\mathrm{x}, \mathrm{y})}{\mathrm{S}^{0^{\circ}}(\mathrm{x}, \mathrm{y})}\right)
$$

Since both the $0^{\circ}$ and the $-90^{\circ}$ signal are modulated by the same local emissivity, the influence of emissivity cancels in (1). This is the basic advantage of displaying the phase signal. In the position of a point heat source the $0^{\circ}$ signal strongly dominates over the $-90^{\circ}$ signal, hence the phase signal in this position is zero and drops linearly to negative values (delay) with increasing distance. This behaviour is independent of the power of the heat source, hence in the phase image weak point heat sources appear with a brightness comparable to strong 
sources, with only a difference in signal-to-noise ratio. This "dynamic compression" feature of the phase signal has already been mentioned to be advantageous for detecting weak heat sources near strong sources [1]. On the other hand, the display of the phase signal also has some disadvantages. It is shown in Fig. 2 that the $0^{\circ}$ signal shows local heat sources with best spatial resolution, since this signal actually diverges for a point heat source at the surface and drops with the inverse of the distance to the source [2]. On the contrary, the $-90^{\circ}$ signal has only a single maximum in the source position and drops much more slowly with increasing distance, hence this signal displays local heat sources with a considerably poorer spatial resolution. Also, the phase signal has only a single maximum in the source position (value zero) and drops slowly (linearly) with increasing distance, hence it also displays heat sources with poor spatial resolution as will be demonstrated below. Herewith, we propose to display the $0^{\circ} / 90^{\circ}$ signal as an alternative to the phase signal:

$$
\mathrm{S}^{0^{\circ} /-90^{\circ}}(\mathrm{x}, \mathrm{y})=\frac{\mathrm{S}^{0^{\circ}}(\mathrm{x}, \mathrm{y})}{\mathrm{S}^{-90^{\circ}}(\mathrm{x}, \mathrm{y})}
$$

This signal is also inherently emissivity-corrected, but contrary to the phase signal (1) this signal also diverges for a point heat source at the surface. Hence, the basic advantage of this new representation of lock-in thermography data is that this signal combines the good spatial resolution of the $0^{\circ}$ signal with the emissivity-corrected property of the phase signal.

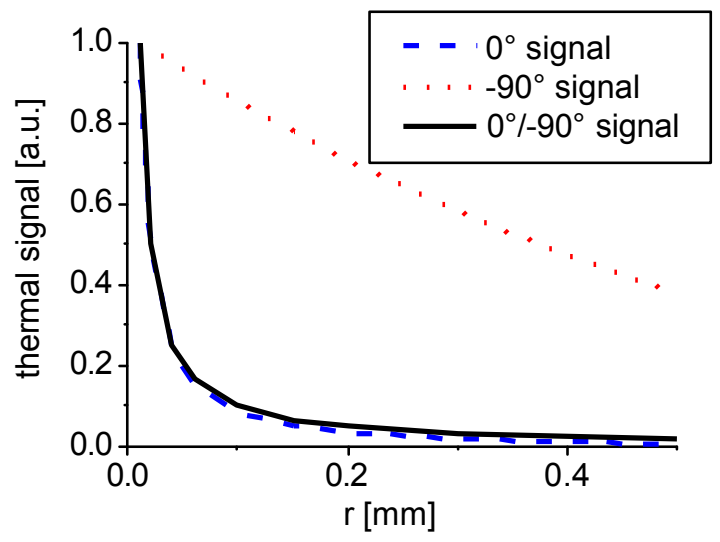

Figure 2: Radial profiles of the $0^{\circ}$ signal, the $-90^{\circ}$ signal, and the $0^{\circ}-90^{\circ}$ signal for a point source at the surface at $r=0$ modulated at $88 \mathrm{~Hz}$. All profiles are separately scaled at $r=$ $10 \mu \mathrm{m}$.

\section{Mathematical deconvolution of the $0 \%-90^{\circ}$ signal}

Another advantage of the $0 \%-90^{\circ}$ signal is that it is the only lock-in thermography signal allowing spatial deconvolution processing in the presence of emissivity contrast. Deconvolution mathematically corrects the usually blurred appearance of lock-in thermography images, which is due to lateral heat conduction within the sample. This "deconvolution" procedure (also called "inversion") inevitably degrades the signal-to-noise ratio of the image, hence only high quality images are suitable for deconvolution. Other presuppositions for spatial image deconvolution are (a) the complete absence of emissivity contrast, and (b) that the signal is a linear superposition of contributions stemming from neighbouring local heat sources. The $0^{\circ}$ signal as well as the $90^{\circ}$ signal and the amplitude signal are all modulated by the local emissivity, hence they all do not fulfill condition (a). The phase signal fulfills condition (a) but not condition (b). If we are working in microscopic dimensions, however, the $0 \%-90^{\circ}$ signal (2) also at least approximately fulfills condition (b). As Fig. 2 shows, compared to the $0^{\circ}$ signal, the $-90^{\circ}$ signal is nearly constant within small distances from the heat source, and the $0^{\circ} /-90^{\circ}$ signal has a very similar shape as the $0^{\circ}$ signal. Thus, it can be taken as an "emissivity-corrected" $0^{\circ}$ signal. Hence, this image can be spatially deconvoluted by assuming it were a $0^{\circ}$ image without any emissivity contrast. We have developed comprehensive lock-in thermography deconvolution / simulation software called "DECONV", which allows the user to perform the spatial deconvolution of different types of lock-in thermography results according to different strategies using a wide range of parameters.

\section{Result of blackening the IC surface}

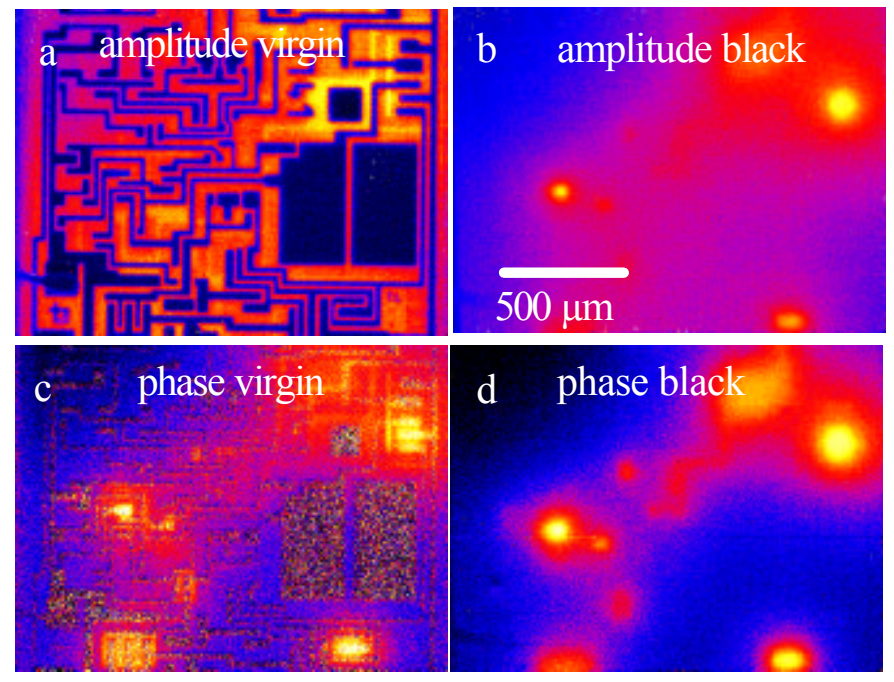

Figure 3: Amplitude images (a) and (b) and phase images (c) and (d) of an IC measured at $24 \mathrm{~Hz}$ without surface coating (a) and (c) and coated with black colloidal bismuth (b) and (d)

Fig. 3 shows lock-in thermograms (amplitude and phase images) of an IC ( $\mu \mathrm{A} 741)$ without and with a colloidal bismuth black surface layer. Here the measuring time was only 5 minutes in both cases. Note that this IC does not contain any fault and is dissipating about $12 \mathrm{~mW}$ in this case, so the images reflect the power dissipated by the normal operation of the IC. Without surface coating for this short measuring time the signal in some metallized regions was so weak that the phase signal shows only noise. The local heat source in the left part of the image is visible only in the phase image but not in the amplitude image. The reason for this is that this heat source lies below a metallized region, hence its signal is 
suppressed and the emissivity contrast dominates the image. After covering the surface with colloidal bismuth (Figs. $3 \mathrm{~b}$ and d), the emissivity contrast in the amplitude image is completely removed and the signal height in the formerly dark (metallized) regions has increased by approximately a factor of 10 . Now all heat sources are also visible in the amplitude signal, and the phase signal shows meaningful values everywhere. On the other hand, the spatial resolution of the blackened sample seems to be slightly degraded, which is probably due to the finite thickness of the black layer.

\section{Synchronous undersampling}

Fig. 4 shows the comparison of amplitude and phase images of the IC $\mu \mathrm{A} 741$ measured with our hitherto maximum squared frame frequency of $45 \mathrm{~Hz}$ compared to those measured by using undersampling at $360 \mathrm{~Hz}$. In both cases the measuring time was $18 \mathrm{~min}$. The red lines in (c) are not artifacts but are real heat sources coming from Joule heating. They are especially clear in the phase image, since this representation displays weak heat sources with stronger contrast $[1,2]$. In the images (b) and (d) taken at $360 \mathrm{~Hz}$ the signal is more confined to the positions of the heat sources, but the noise level is also clearly higher.
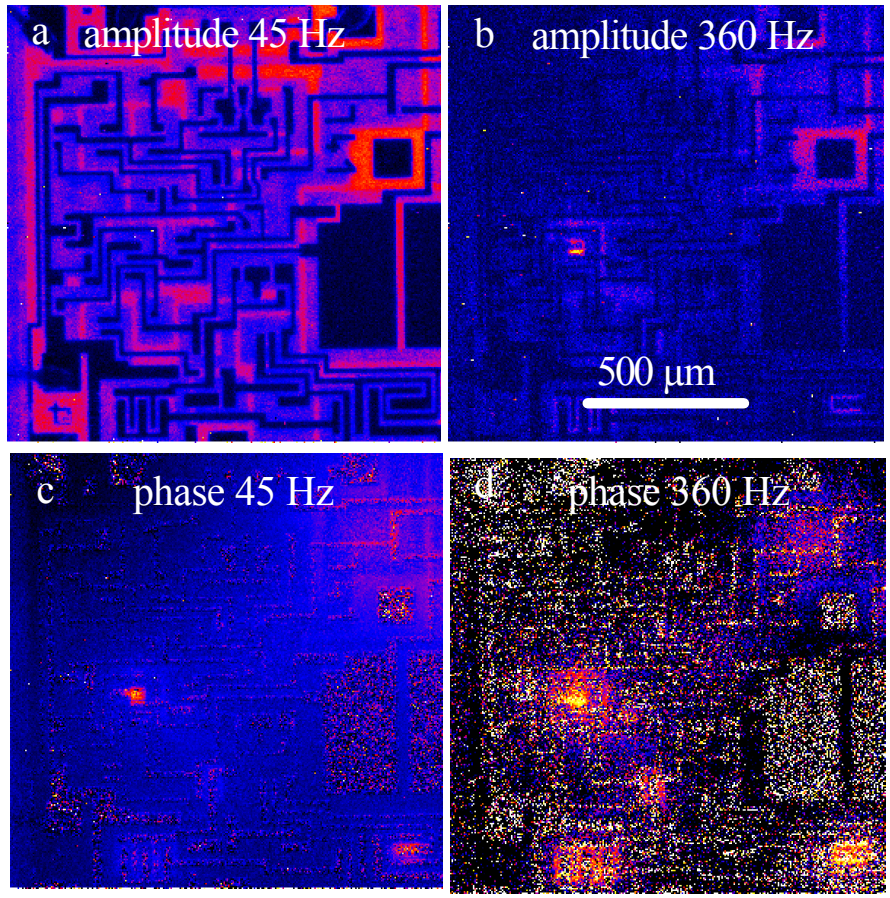

Figure 4: Amplitude images ( $a$ and b) and phase images (c and d) of an IC dissipating $12 \mathrm{~mW}$ measured at $35 \mathrm{~Hz}$ (a and c) and $510 \mathrm{~Hz}$ (b and d) using undersampling

\section{Display of the $0^{\circ} /-90^{\circ}$ signal}

Figs. 5 (a) and (b) show the $0^{\circ}$ and $-90^{\circ}$ images of another IC containing six capacitors (the 6 dark square regions), one of which contains a shunt. These images were taken using an applied voltage of $0.1 \mathrm{~V}$ pulsed at $88 \mathrm{~Hz}$, where a leakage current of about $9.6 \mathrm{~mA}$ was flowing across the shunt. To obtain a very good signal-to-noise ratio, a measuring time of several hours was used. The dominant heat source clearly visible in the $0^{\circ}$ image is not the position of the shunt itself (which is expected to lie in one of the 6 squared regions) but instead a high resistance point in the current path to the shunt. In the $-90^{\circ}$ image the emissivity contrast dominates, as expected, and even this heat source is only weakly visible. Figs. 5 (c) and (d) show the phase image and the $0 \%-90^{\circ}$ image of this region. In both images, there is no emissivity contrast, but the spatial resolution is very different. In the phase image (c), some other heat sources are visible with a comparable brightness except the dominant heat source. These arise from the current leads to the shunt and also the shunt itself shown by the arrow. However, due to the properties of the phase image discussed above, all these heat sources appear very blurred and with comparable brightness. In the $0 \%-90^{\circ}$ image, on the other hand, in addition to the dominant heat source another point heat source is clearly visible in the position of the arrow, which represents the shunt of interest in one of the capacitors.
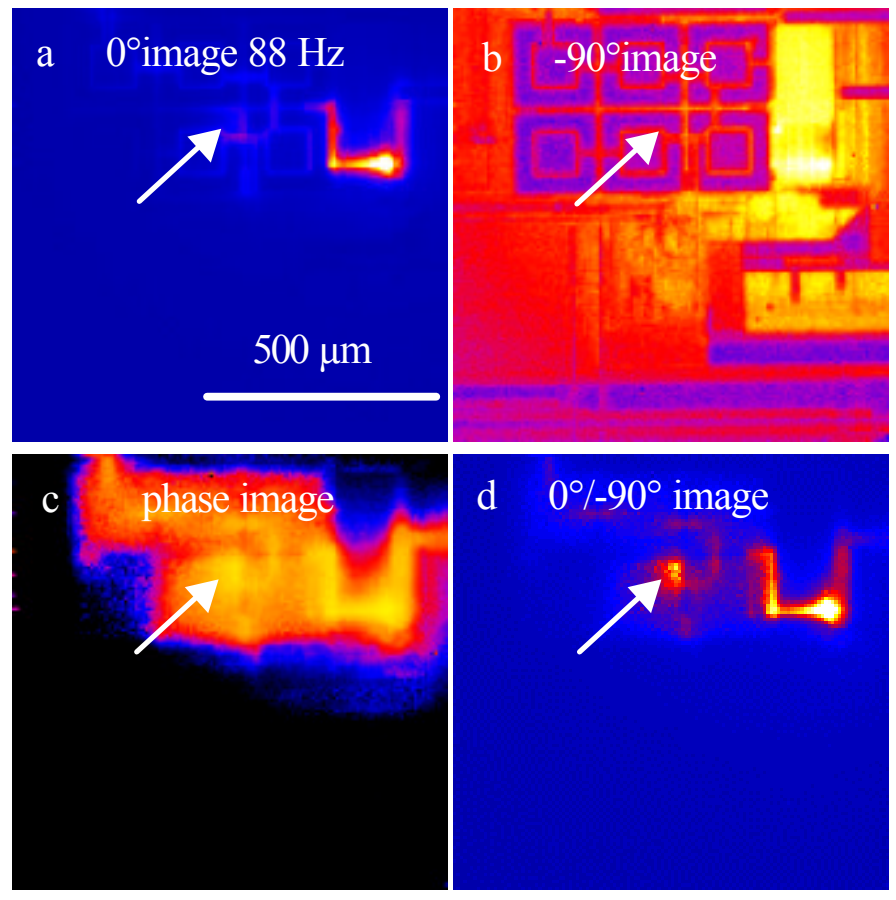

Figure 5: $0^{\circ}$ image (a), $-90^{\circ}$ image (b), phase image (c), and $0 \%-90^{\circ}$ image (d) of a region containing a capacitor shunt (arrows), measured at $88 \mathrm{~Hz}$

\section{Mathematical deconvolution of the $0 \%-90^{\circ}$ signal}

The $0 \%-90^{\circ}$ image shown in Fig. 5 (d) was deconvoluted using our DECONV software, leading to the lateral power distribution shown in Fig. 6. The deconvolution procedure used here was a scalar Fast Fourier Transform (FFT) based procedure using the $0^{\circ}$ point spread function to describe the blurring and Wiener filtering to decrease the influence of the residual noise [2]. In this image the position of a single shunt (arrow) can be defined up to the accuracy of one pixel, which in this case has a width of $7 \mu \mathrm{m}$. On the other hand, the local heat sources are embedded within a certain noise floor, which can be expected for any kind of deconvolution. 


\section{Conclusions and Acknowledgements}

A number of new techniques for performing microscopic lockin thermography failure analysis on ICs have been introduced. These new techniques aim to improve the detection sensitivity (blackening the IC surface) and to improve the effective spatial resolution (the other methods presented). Of course, for an averaging technique like lock-in thermography the sensitivity can always be improved by increasing the measurement time over which the results are averaged. However, by this measure the signal-to-noise ratio (SNR) improves only with $\sqrt{ }_{t_{\text {measure }}}$. Hence, for improving the SNR by a factor of 10 (which is a typical value of the signal degradation for heat sources below metallized layers) a 100-fold measuring time increase is needed compared to the time needed for a similar defect lying below a region of high emissivity. Thus, if very weak heat sources below metallized layers are to be investigated, it may be easier to blacken the surface with colloidal bismuth, as described in this contribution. It depends on the technical possibilities of the user and also on the SNR of the investigated signal, which method is most appropriate to improve the spatial resolution. Displaying the $0 \%-90^{\circ}$ image is certainly the easiest method that is accessible to everyone. The standard edition of the TDL 384 M 'Lock-in' thermography system [4] currently allows to display the $0 \%-90^{\circ}$ image alternatively to the phase image. If the SNR of the measurement is good enough, hence if sufficiently strong heat sources are imaged (beginning at a few $\mathrm{mW}$ ), increasing the lock-in frequency by using undersampling provides an additional possibility to improve the effective spatial resolution. Of course, the $0 \%-90^{\circ}$ signal can also be displayed for images measured in the undersampling mode, hence two of the measures described here can be combined. The TDL 384 M 'Lock-in' system may also be equipped with the undersampling option described here. Alternatively, if the $0 \%-90^{\circ}$ image still shows a very good SNR, its effective spatial resolution can be further improved by mathematical deconvolution. The code of the DECONV software used here is available from the main author, please contact breiten@mpihalle.de. It remains to be systematically investigated whether undersampling or deconvolution leads to better SNR when used for improving the effective spatial resolution of lock-in thermography results.

The authors are indebted to Thermosensorik Erlangen GmbH for continuous and fruitful cooperation in further developing the lock-in thermography technique.

\section{References}

[1] Breitenstein, O., Rakotoniaina, J.P., Altmann, F., Schulz, J., and Linse, G., "Fault Localization and Functional Testing of ICs by Lock-in Thermography", Proc. ISTFA 2002, Phoenix, USA., pp. 29-36.

[2] Breitenstein, O. and Langenkamp, M., Lock-in Thermography - Basics and Use for Functional
Diagnostics of Electronic Components, Springer (Berlin, 2003), ISBN 3-540-43439-9.

[3] Krapez, J.C., "Compared Performance Algorithms Used for Modulation Thermography", Proc. Quantitative Infrared Thermography (QIRT'98), Lodz, Poland, 1998, pp. 148-153.

[4] www.thermosensorik.de

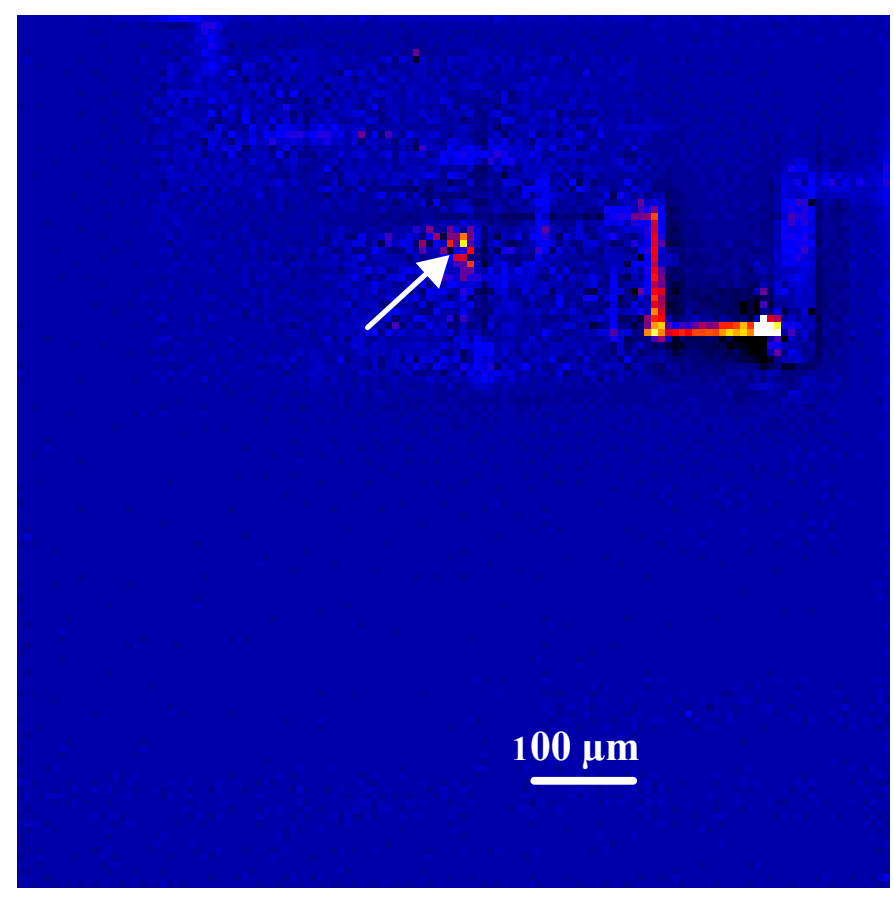

Figure 6: Lateral power distribution obtained from the $0 \%-90^{\circ}$ image in Fig, 5 (d) by mathematical deconvolution. The local shunt (arrow) can be localized to an accuracy of 1 pixel $(7 \mu \mathrm{m})$ 